\title{
Candida albicans blastoconidia in peripheral blood smears from non-neutropenic surgical patients
}

\author{
Y Berrouane, H Bisiau, F Le Baron, C Cattoen, P Duthilleul, E Dei Cas
}

Immunologie et

Cytogénétique

$\mathrm{H}$ Bisiau

F Le Baron

P Duthilleul

Laboratoire de Parasitologie Mycologie, Centre

Hospitalier Régional

Universitaire de Lille,

Lille, France

E Dei Cas

Correspondence to: Dr Y Berrouane, CHRU de Lille, Hôpital A Calmette, Laboratoire de

Bactériologie-Hygiène, $\mathrm{Bd}$ du Pr J Leclercq, 59037 Lille Cédex, France; email: yberrouane@chru-lille.fr

Accepted for publication 19 March 1998

Keywords: blood smear; Candida diagnosis; surgery

Many studies have underlined the recent increase in fungal nosocomial infections. ${ }^{1}$ Predisposing factors for systemic candidiasis include neutropenia, indwelling catheters, multiple antibiotic treatment, and intra-abdominal surgery. ${ }^{1}$ In addition, various fungal infections have been diagnosed by direct detection of fungi in peripheral blood smears. ${ }^{2-8}$ Most patients were immunocompromised. We report here the detection of Candida albicans in a blood smear from a surgical patient.

\section{Case report}

An 80 year old woman was admitted to the general surgery service of the Hospital of

\begin{abstract}
Centre Hospitalier de Valenciennes, Valenciennes, France: Département de Microbiologie et Biochimie

Y Berrouane

C Cattoen

Département

d'Hématologie,

Abstract

An 80 year old woman developed fever 11 days after volvulus surgery. A peripheral blood smear showed numerous yeast cells-both extraleucocytic and intraleucocytic-as well as leucoagglutination. The fungal elements included blastospores, pseudohyphae, and germ tubes. Two days later, blood cultures yielded Candida albicans, Enterobacter aerogenes, and Staphlococcus aureus. The patient had no medical history of immunodeficiency. Several reports indicate that fungal elements may be detected in peripheral blood smears from patients who have a severe intestinal disease. (F Clin Pathol 1998;51:537-538)
\end{abstract} Valenciennes, France, because of an intestinal

Figure 1 Photomicrograph of a May-Grumwald-Giemsa stained peripheral blood smear showing yeast cells and pseudohyphae within a cluster of damaged cells (magnification, $\times 347)$. obstruction related to a volvulus. She had no history of immunodeficiency. Initial laboratory studies showed a white blood cell count of $19.10 \times 10^{9} /$ litre, haemoglobin concentration $126 \mathrm{~g} /$ litre, and a platelet count of $272 \times$ $10^{9} /$ litre. The patient underwent a sigmoidectomy with colostomy.

Histopathological examination showed incipient necrosis of the colonic wall. The patient was treated with amoxicillin-clavulanic acid and ornidazole.

Eleven days after surgery, her temperature rose to $39.5^{\circ} \mathrm{C}$. Blood samples were taken for laboratory screening. The automated haematology analyser (STKS, Coulter Electronics Inc, Hialeah, Florida, USA) indicated flag errors and leucoagglutination was detected using a counting chamber. As was the usual practice, the patient's blood sample was left at $37^{\circ} \mathrm{C}$ for one hour to rule out temperature and EDTA dependent antibodies. Following this, the leucocyte count was $9.4 \times 10^{9} /$ litre, haemoglobin concentration $136 \mathrm{~g} /$ litre, and the platelet count $154 \times 10^{9} /$ litre. On May-GrumwaldGiemsa stained smears, numerous yeast cells were detected, mostly within white blood cells (figs 1 and 2). Blastospores were associated with pseudohyphae and rare germ tubes, which were consistent with $C$ albicans. Clusters of leucocytes and fungal cells were often seen in the margins of the smear.

Microscopic counting of 500 white blood cells showed the following values: neutrophils $56 \%$, eosinophils $0 \%$, basophils $0.2 \%$, lymphocytes $4.4 \%$, and monocytes $2 \% ; 37.4 \%$ of the cells were morphologically damaged and could not be identified. Only nine per cent of neutrophils were morphologically normal; $67 \%$ of neutrophils showed vacuolisation and $24 \%$ contained yeast cells. Examining 200 white blood cells with phagocytosed yeast cells, we found that $49 \%$ were neutrophils and $51 \%$ were non-identifiable. Fluconazole treatment was begun the same day but the patient developed a septic shock and died 48 hours later. $C$ albicans grew from six aerobic and anaerobic blood cultures (Bactec NR 860 ${ }^{\circ}$, Becton Dickinson, Sparks, Maryland, USA) after a two day incubation. The yeast was associated once with Enterobacter aerogenes and twice with Staphylococcus aureus. A urine sample also yielded $C$ albicans and $E$ aerogenes.

\section{Discussion}

Fungal species detected in blood smears include Histoplasma capsulatum, Cryptococcus neoformans, Penicillium marneffei, $C$ albicans, $C$ parapsilosis, $C$ guillermondii, and Hansenula anomala..$^{2-8}$ Most reports describe patients with 


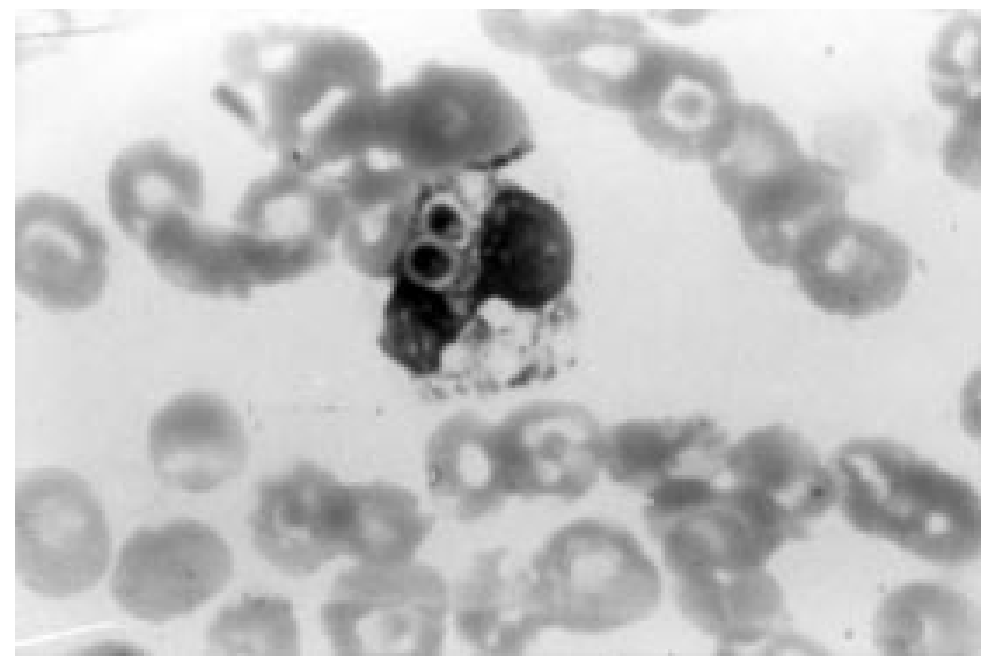

Figure 2 Photomicrograph of a May-Grumwald-Giemsa stained peripheral blood smear showing two yeast cells within a vacuolated neutrophil (magnification, $\times 693$ ).

haematological malignancies or acquired immunodeficiency syndrome..$^{2-48}$ A few other patients have been immunocompetent but they all had an intestinal disease, such as small bowel adhesions, congenital atresia, or carcinoma, most often with intestinal obstruction. ${ }^{5-7}$ Interestingly, $C$ albicans was the species involved in all these cases. In line with these findings, our patient was admitted because of an intestinal obstruction, a necrotising process having already begun at the time of surgery. The fungal elements were so numerous that they were easily detected in stained smears. The development of filament was clearly seen inside neutrophils, suggesting that phagocytosis was not accompanied by fungicidal activity. Although pseudohyphae and true mycelium are typical of the parasitic adaptation of yeast, ${ }^{9}$ they are easily obtained in vitro. Moreover, in vitro phagocytosis of yeasts may occur during a one hour incubation at $37^{\circ} \mathrm{C}$. In the present case, the patient's blood sample was placed at $37^{\circ} \mathrm{C}$ for one hour to rule out artefactual leucoagglutination. ${ }^{10}$ Therefore germination and phagocytosis could have occurred in vitro and not in vivo. However, in other reports, fungi were also phagocytosed, either by neutrophils in the case of Candida sp and P marneffei, or by monocytes in the case of Histoplasma $\mathrm{sp}$, Cryptococcus sp, and $P$ marneffei. ${ }^{2-8}$ Moreover, in candida infected tissues, the neutrophils are the primary inflammatory cell seen and microabscesses are common. Thus in the case described here leucoagglutination was probably the actual host response to fungal invasion and not a laboratory artefact.

Because the inoculum is usually too small for visual detection, diagnosis of fungal bloodstream infections is rarely made by examining peripheral blood smears. Most reports, including ours, indicate that fungal elements are present in large numbers. However, even when the yeast load is high, blood cultures do not yield $C$ albicans before a minimum of two days (range two to five)..$^{5-8}$

Thus leucoagglutination should prompt pathologists to look for yeasts and pseudohyphae in blood smears of patients at risk, such those with severe intestinal lesions.

1 Pfaller MA. Nosocomial candidiasis: emerging species, reservoirs, and modes of transmission. Clin Infect Dis 1996;22 (suppl 2):S89-94.

2 Bonner JR, Alexander WJ, Dismukes WE, et al. Disseminated histoplasmosis in patients with the acquired immune deficiency syndrome. Arch Intern Med 1984;144:2178-81.

3 Yao JDC, Arkin CF, Doweiko JP, et al. Disseminated cryptococcosis diagnosed on peripheral blood smear in a patient with acquired immunodeficiency syndrome. $\mathrm{Am} \mathcal{F} \mathrm{Med}$ 1990;89:100-2.

4 Supparatpinyo K, Sirisanthana T. Disseminated Penicillium marneffei infection diagnosed on examination of a periph-
eral blood smear of a patient with human immunodefieral blood smear of a patient with human immunode
ciency virus infection. Clin Infect Dis 1994;18:246-7.

5 Portnoy J, Wolf PL, Webb M, et al. Candida blastospores and pseudohyphae in blood smears. $N$ Engl $f \mathrm{Med}$ 1971;285:1010-1.

6 Silverman EM, Norman LE, Goldman RT, et al. Diagnosis of systemic candidiasis in smears of venous blood stained with Wright's stain. Am $\mathcal{F}$ Clin Pathol 1973;60:473-5.

7 Kobza K, Steenblock U. Demonstration of Candida in blood smears. BMF 1977;i:1640-1.

8 Girmenia C, Jaalouk G. Detection of Candida in blood smears of patients with hematologic malignancies. Eur $\mathcal{F}$ Haematol 1994;52:124-5.
Hars

9 Dei Cas E, Vernes A. Parasitic adaptation of pathogenic fungi to mammalian hosts. CRC Crit Rev Microbiol 1986;13:173-218.

10 Hillyer CD, Knopf AN, Berkman EM. EDTA-dependent leukoagglutination. Am f Clin Pathol 1990;94:458-61. 